\title{
A REMARK ON THE MOMENT MAP
}

\author{
H. AZAD
}

\author{
(Communicated by Peter Li)
}

\begin{abstract}
Following S. Lie, it is proposed that moment maps be constructed for degenerate forms and the utility of such maps is illustrated by computing the moment maps for torus actions on $\mathbf{C}^{n}$ and $U(n)$ actions on $\mathbf{P}^{n}(\mathbf{C})$.
\end{abstract}

The moment map is usually defined on manifolds with a given nondegenerate 2-form. However, it can also be defined for manifolds with an invariant 2 -form, degenerate or not, and in some sense this useful generalization was already known to Lie. According to Weinstein $[8,2]$, Lie considered the following closely related situation. $M$ is a manifold on which a Lie group $G$ operates, and $\eta$ is a $G$-invariant 1 -form. For $X$ in the Lie algebra $\mathscr{G}$ of $G$ let $X_{M}$ be the corresponding fundamental vector field, i.e., $X_{M}(x)=\left.(d / d t)\right|_{t=0} e^{t X} \cdot x(x \in M)$. Lie proves that the map $\mu: M \rightarrow \mathscr{G}^{*}$ defined by $\mu(x)(X)=\eta_{x}\left(X_{M}(x)\right)(x \in M)$ is $G$-equivariant, with $G$ operating on $\mathscr{G}^{*}$ by the coadjoint representation. Consider the map $\lambda: \mathscr{G} \rightarrow C^{\infty}(M)$ defined by $\lambda(X)=\eta\left(X_{M}\right)$. We have $\mu(x)(X)=\lambda(X)(x)(x \in M, X \in \mathscr{G})$. Now for $Z=X_{M}$ the Lie derivative $L_{Z} \eta$ of $\eta$ along $Z$ is zero, and therefore

$$
0=L_{Z}(\eta)=d(\eta(Z))+i_{Z}(d \eta)
$$

where $i_{Z}$ is the interior product with $Z$ [1]. Now $\omega=d \eta$ is also $G$-invariant and $i_{X_{M}}(\omega)=-d(\lambda(X))$. This leads to the following definition of the comoment map, which, according to [4] was already known to Souriau [7].

Definition (Souriau). Let $M$ be a manifold on which a Lie group $G$ operates and $\omega$ a $G$-invariant 2 -form. Then a comoment for the action of $G$ on $M$ is a linear map $\lambda: \mathscr{G} \rightarrow C^{\infty}(M)$ such that $d(\lambda(X))=-i_{X_{M}}(\omega)$ for all $X \in \mathscr{G}$.

The corresponding moment map is the map $\mu: M \rightarrow \mathscr{G}^{*}$ defined by $\mu(x)(X)$ $=\lambda(X)(x)$, where $x \in M, X \in \mathscr{G}$.

Now our remark is the following proposition which simplifies many of the computations in the literature relating to the moment map.

Proposition. Let $G$ be a Lie group which operates on manifolds $M$ and $\widetilde{M}$, and let $\pi: \widetilde{M} \rightarrow M$ be a G-equivariant map. Assume that $G$ preserves a 2 -form $\omega$ on $M$ and that there is a comoment for the action of $G$ on $M$ with $\mu$ the

Received by the editors November 13, 1992.

1991 Mathematics Subject Classification. 22E70. 
corresponding moment map. Then $\mu \circ \pi$ is a moment map for $\left(\widetilde{M}, \pi^{*}(\omega)\right)$, and if $\tilde{\mu}$ is any other moment map for $\left(\widetilde{M}, \pi^{*}(\omega)\right)$ then $\tilde{\mu}-(\mu \circ \pi)$ is a constant in the sense that for all $x \in M$ and $X \in \mathscr{G}$ we have

$$
\tilde{\mu}(x)(X)-(\mu \circ \pi)(x)(X)=a(X)
$$

for some linear function $a$ on $\mathscr{G}$.

Proof. Let the comoments for $\mu$ and $\tilde{\mu}$ be $\lambda$ and $\tilde{\lambda}$, respectively. For $X \in \mathfrak{g}$ we compare the functions $\pi^{*}(\lambda(X))$ and $\tilde{\lambda}(X)$. We have

$$
-d\left(\pi^{*}(\lambda(X))\right)=\pi^{*}(d(\lambda(X)))=\pi^{*}\left(i_{X_{M}}(\omega)\right)
$$

and $-d(\tilde{\lambda}(X))=i_{X_{\tilde{M}}}\left(\pi^{*}(\omega)\right)$. As $\pi$ is $G$-equivariant we have $\pi^{*}\left(i_{X_{M}}(\omega)\right)=$ $i_{\widetilde{M}_{\tilde{M}}}\left(\pi^{*}(\omega)\right)$. Therefore the functions $\tilde{\lambda}(X)$ and $\pi^{*}(\lambda(X))$ have the same differentials, so their difference is a constant, say $a(X)$. Clearly $a$ is a linear function on $\mathscr{G}$.

\section{Applications}

Let us now apply this remark to recover some computations involving the moment map. The first example serves to introduce some notation needed in the subsequent examples.

(a) Let $M$ be $\mathbf{C}^{n}$ with $\omega$ the standard symplectic form

$$
\omega=\sum d x_{j} \wedge d y_{j}
$$

$z_{j}=x_{j}+i y_{j}(j=1, \ldots, n)$ being the coordinates in $\mathbf{C}^{n}$. We have $\omega=$ $\left.\sum i\left(d z_{j} \wedge d \bar{z}_{j}\right)=i \partial \bar{\partial}\left(\|z\|^{2}\right)=d d^{\mathbf{C}}\|z\|^{2}\right)$, where $d^{\mathbf{C}}=(\partial-\bar{\partial}) / 2 i$. Let $\eta=$ $d^{\mathbf{C}}\left(\|z\|^{2}\right)$. So $\eta$ is invariant under $U(n)$, and the comoment for $\left(\mathbf{C}^{n}, \omega\right)$ is the contraction of $\eta$ with the fundamental vector fields of $U(n)$.

(b) Consider the action of $S^{1}$ on $\mathrm{C}$ with weight $\lambda$; that is, $\exp (i \theta) \cdot z=$ $\exp (i \lambda \theta) z$. The fundamental vector field here is $X(z)=i \lambda z$, i.e., $X(z)=$ $i \lambda(\partial / \partial z)-i \lambda(\partial / \partial \bar{z})$. We have $\eta=d^{\mathbf{C}}(z \bar{z})=(\bar{z} d z-z d \bar{z}) / 2 i$ and $\eta(X)(z)=$ $\lambda z \bar{z}$. So the moment map here is $\mu(z)=\lambda z \bar{z}$. Therefore, for an action of $S^{1}$ on $\mathbf{C}^{n}$ of the form

$$
e^{i \theta}\left(z_{1}, \ldots, z_{n}\right)=\left(e^{i \lambda_{1} \theta} z_{1}, \ldots, e^{i \lambda_{n} \theta} z_{n}\right)
$$

the moment map relative to $\omega=d d^{\mathbf{C}}\left(\|z\|^{2}\right)$ is

$$
\mu\left(z_{1}, \ldots, z_{n}\right)=\lambda_{1}\left|z_{1}\right|^{2}+\cdots+\lambda_{n}\left|z_{n}\right|^{2} .
$$

(c) Finally consider $\mathbf{P}^{n}(\mathbf{C})$. Let $G=U(n+1)$. There is, up to a constant, a unique $G$-invariant hermitian metric on $\mathbf{P}^{n}(\mathbf{C})$. This is the Fubini metric on $\mathbf{P}^{n}(\mathbf{C})$; denote it by $g$. The fundamental form of $g$ is the 2-form $\omega$ defined by $\omega(X, Y)=g(J(X), Y), J$ being the complex structure of $\mathbf{P}^{n}(\mathbf{C})$. Let $\pi: \mathbf{C}^{n+1} \backslash 0 \rightarrow \mathbf{P}^{n}(\mathbf{C})$ be the natural map. It is well known that the pullback of $\omega$ is exact; namely, $\pi^{*}(\omega)=d(\eta)$, where $\eta=d^{\mathbf{C}}\left(\log \|z\|^{2}\right)$. We have already seen that a comoment for an exact form is the contraction of the fundamental vector fields with an invariant potential. Therefore, a moment map $\mu$ for $\mathrm{C}^{n+1} \backslash 0$ is

$$
\mu(z)(X)=d^{\mathbf{C}} \log \left(\|z\|^{2}\right)(X)=\left(1 /\|z\|^{2}\right) d^{\mathbf{C}}\left(\|z\|^{2}\right)(X) \quad\left(z \in \mathbf{C}^{n+1} \backslash 0, X \in \mathscr{G}\right) .
$$


The map $\mu$ is $G$-equivariant as $\eta$ is $G$-invariant. Moreover, as $\mu(\lambda z)=\mu(z)$ the map $\mu$ descends to a $G$-equivariant map $\bar{\mu}$ from $\mathbf{P}^{n}(\mathbf{C})$ into $\mathscr{G}^{*}$. By the proposition $\bar{\mu}$ is a moment map for $\mathbf{P}^{n}$ (relative to the Fubini metric).

Ness [6] and Kirwan [5] first guess the formula for $\bar{\mu}$ and then verify that it is indeed a moment map. In a somewhat more general context, in GuilleminSternberg [3] there appears in $\S 3$ a complex line-bundle $L$ over a compact Kähler manifold $X$ so that if $\omega$ is the fundamental form of $X$ then $\pi^{*}(\omega)$ is an exact form with potential, say $\eta$ ( $\eta$ is a connection 1 -form), and from their formulae it is clear that the momentum mapping corresponds to contracting the vector fields on $L$ with $\eta$. We owe this remark to Professor J. J. Duistermaat.

\section{REFERENCES}

1. R. Abraham and J. Marsden, Foundations of mechanics, Addison-Wesley, Reading, MA, 1985.

2. A. M. Bloch and T. S. Ratiu, Convexity and integrability, Symplectic Geometry and Mathematical Physics, Birkhäuser, Basel, 1991, pp. 48-79.

3. V. Guillemin and S. Sternberg, Geometric quantization and multiplicities of group representations, Invent. Math. 67 (1982), 515-538.

4. P. Iglesias, Les $\mathrm{SO}(3)$-variétés symplectiques et leur classification en dimension 4, Bull. Soc. Math. France 119 (1991), 371-369.

5. F. Kirwan, The cohomology of quotients in symplectic and algebraic geometry, Math Notes 31, Princeton Univ. Press, Princeton, NJ, 1985.

6. L. Ness, A stratification of the null-cone via the moment map, Amer. J. Math. 106 (1984), 1281-1329.

7. J. M. Souriau, Structure des systèmes dynamiques, Dunod, Paris, 1970.

8. A. Weinstein, Sophus Lie and symplectic geometry, Exposition. Math. 1 (1983), 95-98.

Mathematisches Institut, Ruhr-Universität, Bochum, Germany

Department of Mathematics, Quaid-i-Azam University, Islamabad, Pakistan 